\title{
Specialty Grand Challenges in Biosensors
}

\author{
Valtencir Zucolotto* \\ Nanomedicine and Nanotoxicology Group, Physics Institute of Sao Carlos, University of São Paulo, São Carlos, Brazil
}

Keywords: biosensor, grand challenge, nanomedicine, nanosensing, device

Since the pioneering work of Clark and Lyons in the 1960s on an analytical enzymatic device capable of detecting glucose (Clark and Lyons, 1962), research on biosensors has experienced tremendous advances. Despite the variety of biomolecules that have been applied both as the biorecognition layer and as analytes, a myriad of biosensors platforms with different applicability have been developed, including wearable, implantable, and most recently, ingestible biosensors. To date, a number of scientific and technological areas have benefited from the development and applications of biosensors, with emphasis on the healthcare and medical areas, food industry, and environmental monitoring. Just like others scientific and technological fields, research on biosensors has benefited enormously from the use of novel materials and technologies, as in the case of nanotechnology, through which more efficient biosensor systems are regularly reported. Among the main nanomaterials reported for application in sensors, gold nanoparticles, quantum dots, carbon nanotubes, and graphenes are the most representative ones (Brazaca et al., 2019). However, despite the numerous scientific and technological advances reported in the area of biosensors, a gap between such advances and the number of products based on biosensors available commercially is clearly observed. Such a gap is probably related the difficulties of manufacturing robust and reliable devices, with good specificity, sensitivity, and above all, reproducibility on a large scale. These challenges apply for all application sectors, including medical applications which, despite having the largest number of commercially available devices, still suffers from a lack of products that can meet the needs of point-of-care applications (portable, low-cost, fast response, disposable).

In the medical field, biosensors have been used for many years, and it is certainly the area that

\section{OPEN ACCESS}

Edited and reviewed by: Dermot Diamond Dublin City University, Ireland

*Correspondence: Valtencir Zucolotto zuco@ifsc.usp.br

Specialty section:

This article was submitted to Biosensors,

a section of the journal Frontiers in Sensors

Received: 05 July 2020

Accepted: 17 July 2020 Published: 20 August 2020

Citation:

Zucolotto V (2020) Specialty Grand Challenges in Biosensors. Front. Sens. 1:3.

doi: 10.3389/fsens.2020.00003 benefits from the largest number of commercial devices, such as the glucometer, a biosensor that revolutionized the monitoring of blood glucose levels, improving the quality of life of millions of diabetic people worldwide. And demand from the biomedical sector is set to rise much further when we consider the impact of "the medicine of the future" or "P4 medicine," that is predictive, personalized, preventative, and participatory (Hood and Friend, 2011)." In this scenario, conceived for future years, POC-type biosensor devices will have a fundamental role, since they can provide real-time monitoring and diagnosis of a series of diseases, through reproducible and reliable measures, carried out by the patient, or in some cases, automatically, through wearable, implantable, or ingestible devices. However, we are not yet in that degree of clinical application for biosensor devices, with some significant obstacles to be overcome, both scientifically and technologically, so that we can have devices produced and used on a large scale.

In addition to the marketing aspects, which we will not deal with here, perhaps the main challenge for biosensors to become key analytical tools for $\mathrm{P} 4$ medicine is the ability to produce large-scale disposable devices at a low cost, and especially with good accuracy and reproducibility. Immunosensors, for example, are biosensors capable of quickly detecting antigens (or antibodies, depending on their configuration) in samples such as serum, plasma, blood, saliva, urine, etc., thus becoming tools of importance for the development of rapid test kits, especially for infectious diseases such as Zika and Dengue diseases, Malaria, and COVID-19, amongst others. Although these devices are already widely used, problems related to low sensitivity and specificity that may provide unacceptable levels of false-positive or false-negative results still represent a major obstacle and a limiting factor that needs optimization. 
Another type of biosensors of biomedical importance, the genosensors-devices capable of detecting a specific sequence of genetic material-offer a relatively simple platform to provide diagnostics with very high accuracy for diagnosing genetic-based diseases, or even to detect the presence of genetic material from pathogens such as viruses and bacteria. These latter biosensors have the potential to revolutionize the field of molecular diagnostics, currently dominated by the polymerase chain reaction (PCR) technology, which despite the great accuracy and efficiency, is time-consuming and requires high-cost equipment and qualified personnel. One of the main challenges and limitations for the full development and manufacture of genosensors lies in the sample preparation step, where isolation and amplification of the genetic material are necessary. However, with the advent of the Loop-mediated isothermal amplification (LAMP) technique, part of these sample preparation limitations could be overcome. In contrast to PCRbased approaches, LAMP devices are capable of amplifying genetic material at constant temperatures, requiring less complex equipment that can be directly coupled to genosensors for the final reading of the amplified material. However, there is still a long way to go in this direction, both from the scientific and technological perspectives.

Perhaps the most recent breakthroughs regarding the use of biosensors in medical fields are the wearable, implantable, and the ingestible biosensor devices. For wearable and implantable devices, in particular, despite the numerous publications reporting their applications in clothing, glasses, or in the form of patches, capable of communicating wirelessly with a smartphone, there is still much work to be done to ensure their long-term stability and operationality. Ingestible biosensors are even more recent, representing perhaps, the state-of-the-art in the vast field of biosensors research, and the future of personalized medicine (Mimee et al., 2018). These swallowable devices are capable of monitoring in real time a series of parameters inside the human body, especially in the digestive tract, transferring the data to a remote monitoring system, wirelessly. Ingestible biosensors are currently hot topics in this sub-area of convergence that encompasses biotechnology, analytical chemistry, microelectronics and signal processing, and for this reason, important challenges must be overcome until the complete implementation of these systems, in the form of reliable, accurate, and highly secure medical microdevices.

Despite the medical areas, where the application of commercial biosensors is already underway, areas such as environmental monitoring, agriculture, and the food industry also benefit from the use of biosensors. Devices for monitoring chemical pollutants, for example, in water or in the ground have been reported. Despite their efficiency, one of the main challenges to be overcome by such devices is related to their robustness for outdoor use. Facing similar challenges are the biosensors for use in precision agriculture, which must also be robust enough to keep fully operational in the field, even for extended periods. These devices are of great interest in agricultural areas, with applications ranging from the monitoring of crop pests and plants diseases, to the analysis of chemical and biological components in the soil. Their integration into a practical information and control system through wireless monitoring, for example, is a crucial factor to be developed prior to their application in the so-called digital farms.

Finally, rapid detection devices represent a major breakthrough in the areas of food processing, especially for food quality control. The development and production of devices for monitoring chemical or biological contaminants in food represents a challenge for such applications, where selectivity and specificity must be the main parameters to be controlled and optimized.

In conclusion, despite the very encouraging scenario regarding the application of biosensors as analytical devices in strategic scientific and technological areas, limitations still persist, representing great challenges in the field. Advances related to one or more of the challenges listed below are more than welcome in the field of biosensing:

- Optimizing accuracy, sensitivity, and specificity of biosensors for serological rapid tests, specifically for infectious diseases;

- Improving reproducibility and scalability of manufacturing POC biosensor devices;

- Pushing down the limit of detection of POC biosensors for cancer and cardiac biomarkers detection;

- Optimizing the integration between genosensors and LAMP platforms, capable of simplifying the sample preparation steps, providing rapid genetic tests;

- Improving the efficient manufacture of disposable, low cost, and high efficiency biosensor devices;

- Integrating biochemical recognition layers and nanomaterials in detection platforms;

- Optimizing platforms and improving the performance of wearable biosensors;

- Optimizing the development of ingestible biosensors integrated with electronic devices for in vivo monitoring in real time;

- Developing accurate and robust biosensors integrated with remote control systems for environmental monitoring;

- Improving the robustness and accuracy of biosensors for agricultural application and quality control in the food industry.

\section{AUTHOR CONTRIBUTIONS}

The author confirms being the sole contributor of this work and has approved it for publication. 


\section{REFERENCES}

Brazaca, L. C., Moreto, J. R., Martín, A., Tehrani, F., Wang, J., Zucolotto, V. (2019). Colorimetric paper-based immunosensor for simultaneous determination of fetuin B and clusterin toward early Alzheimer's diagnosis. ACS Nano 13, 13325-13332. doi: 10.1021/acsnano.9b06571

Clark, L. Jr., and Lyons, C. (1962). Electrode systems for continuous monitoring in cardiovascular surgery. Ann. N. Y. Acad. Sci. 102:29. doi: 10.1111/j.1749-6632.1962.tb1 3623.x

Hood, L., and Friend, S. H. (2011). Predictive, personalized, preventive, participatory (P4) cancer medicine. Nat. Rev. Clin. Oncol. 8, 184-187. doi: 10.1038/nrclinonc.20 10.227
Mimee, M., Nadeau, P., Hayward, A., Carim, S., Flanagan, S., Jerger, L., et al. (2018). An ingestible bacterial-electronic system to monitor gastrointestinal health. Science 360, 915-918. doi: 10.1126/science.aas9315

Conflict of Interest: The author declares that the research was conducted in the absence of any commercial or financial relationships that could be construed as a potential conflict of interest.

Copyright $\odot 2020$ Zucolotto. This is an open-access article distributed under the terms of the Creative Commons Attribution License (CC BY). The use, distribution or reproduction in other forums is permitted, provided the original author $(s)$ and the copyright owner(s) are credited and that the original publication in this journal is cited, in accordance with accepted academic practice. No use, distribution or reproduction is permitted which does not comply with these terms. 